\title{
A single-photon server with just one atom
}

\author{
MARKUS HIJLKEMA ${ }^{1}$, BERNHARD WEBER ${ }^{1}$, HOLGER P. SPECHT ${ }^{1}$, SIMON C. WEBSTER ${ }^{1}$, AXEL KUHN² \\ AND GERHARD REMPE ${ }^{1 *}$
}

\author{
${ }^{1}$ Max-Planck-Institut für Quantenoptik, Hans-Kopfermann-Str. 1, D-85748 Garching, Germany \\ ${ }^{2}$ Department of Physics, University of Oxford, Clarendon Laboratory, Parks Road, Oxford OX1 3PU, UK \\ *e-mail: gerhard.rempe@mpq.mpg.de
}

Neutral atoms are ideal objects for the deterministic processing of quantum information. Entanglement operations have been carried out by photon exchange ${ }^{1}$ or controlled collisions ${ }^{2}$, and atom-photon interfaces have been realized with single atoms in free space $^{3,4}$ or strongly coupled to an optical cavity ${ }^{5,6}$. A long-standing challenge with neutral atoms, however, is to overcome the limited observation time. Without exception, quantum effects appeared only after ensemble averaging. Here, we report on a single-photon source with one, and only one, atom quasi-permanently coupled to a high-finesse cavity. 'Quasipermanent' refers to our ability to keep the atom long enough to, first, quantify the photon-emission statistics and, second, guarantee the subsequent performance as a single-photon server delivering up to 300,000 photons for up to $30 \mathrm{~s}$. This is achieved by a unique combination of single-photon generation and atom cooling ${ }^{7-9}$. Our scheme brings deterministic protocols of quantum information science with light and matter ${ }^{10-16}$ closer to realization.

Deterministic single-photon sources are of prime importance in quantum information science ${ }^{17}$. Such sources have been realized with neutral atoms, embedded molecules, trapped ions, quantum dots and defect centres $^{18}$. All of these sources are suitable for applications where the indivisibility of the emitted light pulses is essential. For quantum computing or quantum networking, the emitted photons must also be indistinguishable. Such photons have so far only been produced with quantum $\operatorname{dots}^{19}$ and atoms $s^{20,21}$. Another requirement is a high efficiency. This is hard to obtain in free space, as the light-collecting lens covers only a fraction of the full $4 \pi$ solid angle. The efficiency can be boosted by strongly coupling the radiating object to an optical microcavity, as has been achieved with atoms $s^{5,6}$ and quantum $\operatorname{dots}^{22}$. An additional advantage of the cavity is that a vacuum-stimulated Raman adiabatic passage can be driven in a multilevel atom ${ }^{6,23,24}$. In this way, the amplitude $e^{5,24}$, frequency ${ }^{20}$ and polarization ${ }^{25}$ of the photon can be controlled. It should also be possible to combine partial photon production with internal atomic rotations for the construction of entangled photon states such as $\mathrm{W}$ and GHZ states ${ }^{15}$.

All of these demands together have so far only been achieved with atoms in high-finesse microcavities. One reason is that neutral atoms are largely immune to perturbations, such as electric patch fields close to dielectric mirrors. However, atomic systems have always suffered from a fast atom loss. We have now implemented a cavity-based scheme, see Fig. 1, with a dipole laser for trapping, a trigger laser for photon generation and a recycling laser for

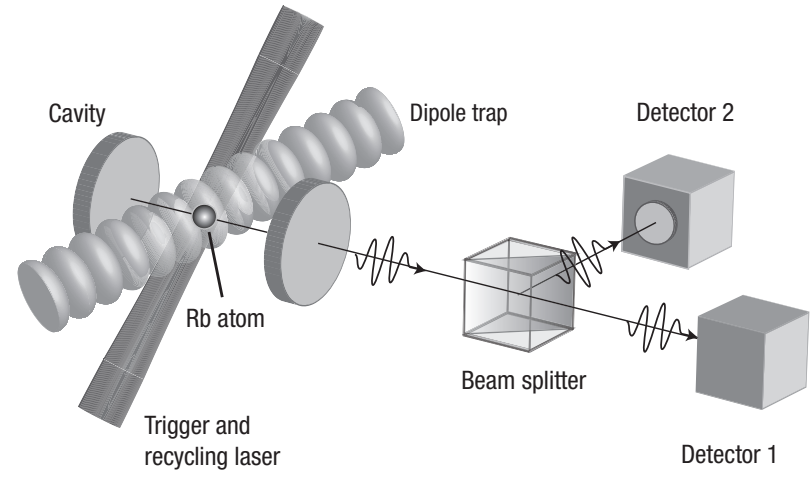

Figure 1 Schematic diagram of the apparatus. A single ${ }^{85} \mathrm{Rb}$ atom is trapped in a high-finesse optical microcavity by means of a two-dimensional optical lattice. Confinement along the cavity axis and a direction perpendicular to it is achieved with a weak cavity-stabilization laser and a strong retroreflected dipole laser, respectively. Confinement along the third direction results mainly from the small beam waist of the dipole laser. The atom-cavity system is excited by a sequence of laser pulses incident under an angle of $45^{\circ}$ to the dipole laser and perpendicular to the cavity axis. Single photons emitted from the system are detected by two avalanche photodiodes in the Hanbury Brown and Twiss configuration. For simplicity, details of the set-up such as the set of prisms and interference filters in front of the detectors are not shown.

repumping, monitoring and cooling the atom ${ }^{8,9}$. The scheme combines high photon-generation efficiency and long trapping times. The most remarkable features are, first, that the singlephoton stream is specified by its intensity correlation function evaluated in real-time during a short time interval after system preparation and, second, that its subsequent performance is guaranteed by monitoring the atom without perturbing the singlephoton stream. This makes our single-photon source a useful quantum device operating with just one atom.

The main parts of the apparatus are described elsewhere $e^{8,26}$, but changes were made to allow for single-photon generation and detection in combination with atom cooling. In short, ${ }^{85} \mathrm{Rb}$ atoms are collected from a background vapour in a magneto-optical trap, loaded into a running-wave dipole trap (wavelength 1,032 $\mathrm{nm}$ ) and transferred into the optical high-finesse cavity. On arrival, a few atoms are captured by switching the geometry of the dipole trap to a standing wave and turning on 780-nm lasers perpendicular 


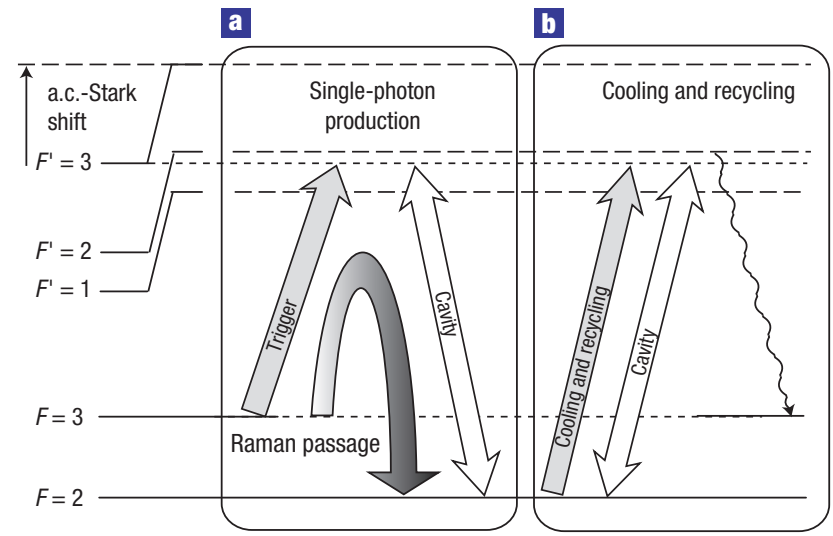

G

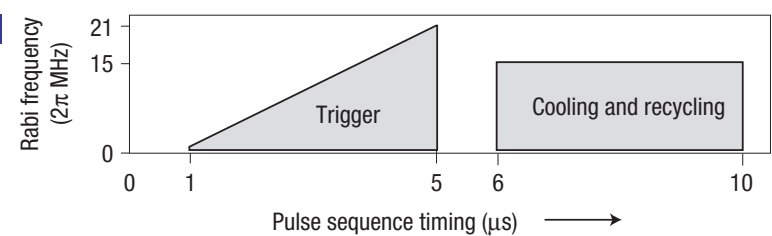

Figure 2 Simplified energy-level diagram. a,b, During single-photon production (a), the ${ }^{85} \mathrm{Rb}$ atom is excited by a 4 - $\mu$ s-long pulse of a trigger laser resonant with the $F=3 \rightarrow F^{\prime}=3$ transition between the $5 \mathrm{~S}_{1 / 2}$ ground and $5 \mathrm{P}_{3 / 2}$ excited state. With the cavity resonant with the $F=2 \rightarrow F^{\prime}=3$ transition, this drives a vacuum-stimulated Raman adiabatic passage from $F=3$ to $F=2$, leading to the emission of a photon from the cavity (b). One microsecond later, a 4- $\mu$ s-long laser pulse is used to recycle the atom back into the $F=3$ state. This laser is resonant with the cavity and the $F=2 \rightarrow F^{\prime}=3$ transition. As the presence of the trapping potential leads to a dynamic energy-level shift (the a.c.-Stark shift) of $\Delta_{\mathrm{S}} \approx 2 \pi \times 70 \mathrm{MHz}, 7 \mathrm{MHz}$ larger than the atomic hyperfine splitting between the $F^{\prime}=2$ and $F^{\prime}=3$ states, all lasers and the cavity are red-detuned from the atomic transitions from the ground states to the $F^{\prime}=2$ excited state by a few $\mathrm{MHz}$. This results in a situation where the atom can cycle a few times, scattering photons into the cavity, before falling back into the $F=3$ state. This cycling cools the atom. c, The timing sequence of the laser pulses with their pulse shapes.

to the cavity axis for three-dimensional cavity cooling ${ }^{8}$. The cavity has a length of $0.5 \mathrm{~mm}$, a mode waist of $29 \mu \mathrm{m}$ and a finesse of $3 \times 10^{4}$. The relevant parameters are $(g, \kappa, \gamma)=2 \pi \times(5,5,3) \mathrm{MHz}$, where $g$ is the maximum atom-cavity coupling constant on the $F=2 \rightarrow F^{\prime}=3$ transition between the atomic $5 \mathrm{~S}_{1 / 2}$ ground and $5 \mathrm{P}_{3 / 2}$ excited state, $\kappa$ is the cavity-field decay rate and $\gamma$ is the atomic dipole decay rate. One of the cavity mirrors has a 50 times higher transmittance than the other. Photons scattered into the cavity by the trapped atom and emitted through this output mirror are spectrally and spatially filtered from the light of a cavitystabilization laser at $785 \mathrm{~nm}$ and stray light, respectively, by means of cascaded glass prisms in combination with interference filters and pinholes. Finally, the photons are counted by two avalanche photodiodes. The combined background count rate due to stray light and dark counts is $84 \mathrm{~Hz}$.

Figure 2 shows the three-level system that enables singlephoton production and atom cooling. Starting with a single atom in the $F=3$ ground state, a trigger pulse together with the cavity drives a vacuum-stimulated Raman adiabatic passage ${ }^{23,27}$ into the $F=2$ ground state (see Fig. $2 \mathrm{a}$ ). This generates a single photon that is emitted from the cavity. Next, the atom is pumped back to the initial $F=3$ state with a recycling laser resonant with the cavity (see Fig. 2b). During this recycling process, the atom can scatter many photons into the cavity. To understand the scattering process in more detail, the $k_{\mathrm{B}} \times 1.5 \mathrm{mK}$ deep dipole trap has to be taken into account ( $k_{\mathrm{B}}$ is Boltzmann's constant). The trap shifts the atomic resonances by $\Delta_{\mathrm{S}} \approx 2 \pi \times 70 \mathrm{MHz}$, the dynamic Stark shift. As shown in Fig. 2a,b, all of the lasers and the cavity are not resonant with the atom. This has little consequence for the photon production in the Raman process as both ground states experience the same shift. For the recycling laser, however, it creates a situation similar to one described earlier, with a strong Sisyphus-like cooling force $^{8,9}$. This occurs even though the atomic transition used is not closed so that cooling takes place only as long as the atom cycles between the ground and excited state. Note that full threedimensional cavity cooling is used only for the initial trapping of the atom. Here, both the trigger laser (now acting as a repumper) and the recycling laser are turned on continuously. This can keep the atom in the cavity for up to $1 \mathrm{~min}$.

While applying the photon-production and recycling pulses, atoms stay in the cavity for 10.3(1) s on average, as determined from 526 experimental runs. This is about twice as long as in the dark dipole-force trap. Taking into account that initially several atoms are trapped and that we have to wait until all but one of the atoms have escaped the cavity, this gives $4,379 \mathrm{~s}$ of singleatom data with $4.23 \times 10^{6}$ detection events during trigger pulses, of which $0.15 \times 10^{6}$ are background detections. Single atoms are available for single-photon production for 8.3(2) s on average. For a trigger rate of $100 \mathrm{kHz}$, the overall photon generation, propagation and detection probability then amounts to $0.93 \%$. This includes $50 \%$ cavity absorption loss mainly due to a mirror defect, $52 \%$ propagation loss from the cavity to the detectors and $44 \%$ quantum efficiency of the detectors. The photon-generation probability is therefore $9 \%$. The finite efficiency is attributed to the large number of Zeeman states, some of them exhibiting a small (for example, $g=2 \pi \times 1.4 \mathrm{MHz}$ ) atom-cavity coupling constant. In addition, because the coupling constant and the dynamic Stark shift are position dependent, the residual motion of the atom in the trap may play a role.

We calculate the cross-correlation of the recorded photon stream binned over 4- $\mu$ s-long intervals corresponding to the trigger pulses (discarding detection events outside the trigger pulse). Summed over the 526 runs, this gives $1.2 \times 10^{4}$ correlations on average for each time bin. This gives 22 correlations per atom per bin. For a pulsed light source, correlations appear periodic with the repetition rate. For single photons equal-time coincidences do not occur. This absence of correlations at $\Delta \tau=0$ is referred to as antibunching. The measured antibunching visibility averaged over the 526 runs is $94.0 \%$.

The large number of correlations observed per atom and the large visibility of the antibunching suggests the following measurement protocol for single-atom operation, see Fig. 3. First, the system is initialized by trapping a few atoms and monitoring the light level emitted from the cavity during recycling pulses. When this level reaches a value expected for one atom ( 4 photons $\mathrm{ms}^{-1}$ on average), the photons emitted during trigger pulses within the next $1.5 \mathrm{~s}$ are recorded and the cross-correlation of the (binned) photon stream is calculated, see Fig. 3a, left inset. Next, the data are tested against the selection rule that the average number of correlations for non-zero time differences must exceed 1.5 (to make sure that at least one atom is trapped, leading to four correlations on average) and that correlations at zero time difference must not exceed $30 \%$ of this average. The latter condition makes sure that not more than one atom is trapped, a conclusion that in our experiment cannot be obtained from the scattering rate alone, as two (or more) weakly coupled atoms could produce the same fluorescence signal as one strongly coupled atom. We find that $86 \%$ of all 526 runs pass such a test. This leaves 454 runs with 3,774 s of true single-photon data with on average $1.0 \times 10^{4}$ correlations at non-zero time difference 

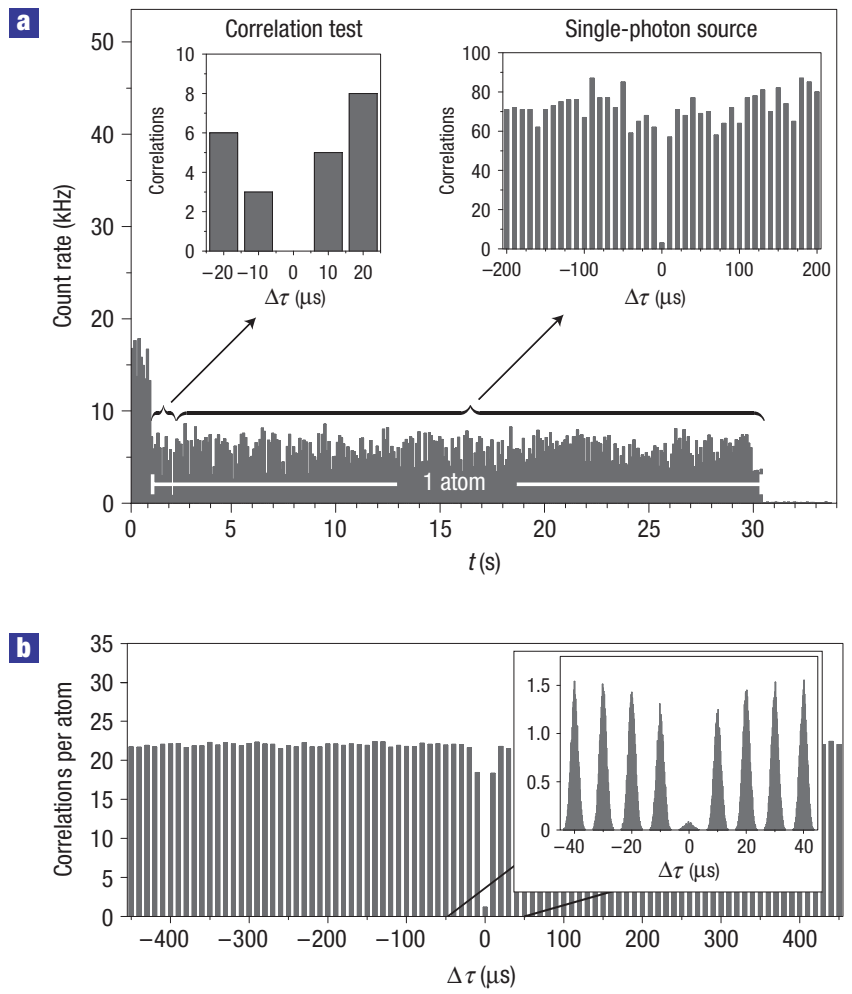

Figure 3 Photon statistics of our single-photon server. a, Performance for just one atom. The light emitted from the cavity during single-photon production and recycling is plotted versus time for one experimental run. Initially, a few atoms are trapped in the cavity. After $1 \mathrm{~s}$, the average count rate drops to the single-atom level. To verify that exactly one atom is trapped, we calculate the cross-correlation of the photons recorded by the two detectors during trigger pulses for the next $1.5 \mathrm{~s}$. A single atom manifests itself by the absence of coincidences for zero detection-time difference, $\Delta \tau=0$, in the correlation function, as shown in the upper left inset. The upper right inset shows the correlation function obtained from the remaining 28 -s-long single-photon stream, containing $\sim 2.8 \times 10^{5}$ single photons, of which we detect $11 \%$. For this specific experimental run, antibunching has a visibility of $95.8 \%$. b, Average behaviour. Correlation function averaged over those 454 single-atom runs that passed the qualification procedure described in the text. Antibunching has a visibility of $94.6 \%$, limited only by background counts. The similarity of the single-atom trace shown in a compared to the average behaviour demonstrates the deterministic character of our source. The inset shows the averaged correlation function with a time resolution of $200 \mathrm{~ns}$. The comb structure is due to the pulsed nature of the experiment.

and 534 coincidences at zero time difference. From our background count rate, we would expect 587(24) coincidences of a photon click with a background click. The measured antibunching with its visibility of $94.6 \%$ is therefore entirely limited by dark counts and stray light.

A single-atom source that passed the described selection procedure therefore emits a high-quality stream of single photons. A user of the photons can be notified and the photons redirected as needed. While this is done, the presence of the atom is monitored by detecting the light emitted during the recycling pulses. A loss of the atom manifests itself by the absence of scattered photons that can be detected within $\sim 30$ ms with $98 \%$ probability. In the right inset of Fig. 3a, the photon correlation function is plotted for those photons that would have been sent to the user. For comparison, the correlation function for all 454 runs is shown in Fig. 3b. The correlation signal obtained for one-and-the-same atom clearly shows the antibunching that is otherwise observed only after averaging over an ensemble of single atoms. Note that in contrast to all previous single-atom experiments, the single-atom nature of our system is obtained from a non-classical correlation signal, not a classical average of the emitted photon stream. This unambiguously discriminates a single atom from several atoms.

In summary, our atom-cavity system has progressed from a proof-of-principle single-photon source to a useful device whose performance is specified during operation. The quasi-permanent availability of exactly one atom, the high efficiency of photon production in a well-defined light mode and the large duty cycle of the whole measurement sequence paves the way for deterministic atom-photon and atom-atom entanglement experiments such as a test of Bell's inequality with distant atoms.

Received 22 December 2006; accepted 8 February 2007; published 11 March 2007.

References

1. Raimond, J. M., Brune, M. \& Haroche, S. Colloquium: Manipulating quantum entanglement with atoms and photons in a cavity. Rev. Mod. Phys. 73, 565-582 (2001).

2. Mandel, O. et al. Controlled collisions for multi-particle entanglement of optically trapped atoms. Nature 425, 937-940 (2003).

3. Darquié, B. et al. Controlled single-photon emission from a single trapped two-level atom. Science 309, 454-456 (2005).

4. Volz, J. et al. Observation of entanglement of a single photon with a trapped atom. Phys. Rev. Lett. 96, 030404 (2006).

5. Kuhn, A., Hennrich, M. \& Rempe, G. Deterministic single-photon source for distributed quantum networking. Phys. Rev. Lett. 89, 067901 (2002).

6. McKeever, J. et al. Deterministic generation of single photons from one atom trapped in a cavity. Science 303, 1992-1994 (2004).

7. Maunz, P. et al. Cavity cooling of a single atom. Nature 428, 50-52 (2004).

8. Nußmann, S. et al. Vacuum-stimulated cooling of single atoms in three dimensions. Nature Phys. 1, 122-126 (2005).

9. Murr, K. et al. Three-dimensional cavity cooling and trapping in an optical lattice. Phys. Rev. A 73, 063415 (2006).

10. Cirac, J. I., Zoller, P., Kimble, H. J. \& Mabuchi, H. Quantum state transfer and entanglement distribution among distant nodes in a quantum network. Phys. Rev. Lett. 78, 3221-3224 (1997).

11. Pellizzari, T. Quantum networking with optical fibres. Phys. Rev. Lett. 79, 5242-5245 (1997).

12. Browne, D. E., Plenio, M. B. \& Huelga, S. F. Robust creation of entanglement between ions in spatially separate cavities. Phys. Rev. Lett. 91, 067901 (2003).

13. Clark, S., Peng, A., Gu, M. \& Parkins, S. Unconditional preparation of entanglement between atoms in cascaded optical cavities. Phys. Rev. Lett. 91, 177901 (2003).

14. Duan, L.-M. \& Kimble, H. J. Scalable photonic quantum computation through cavity-assisted interactions. Phys. Rev. Lett. 92, 127902 (2004).

15. Schön, C., Solano, E., Verstraete, F., Cirac, J. I. \& Wolf, M. M. Sequential generation of entangled multiqubit states. Phys. Rev. Lett. 95, 110503 (2005).

16. Serafini, A., Mancini, S. \& Bose, S. Distributed quantum computation via optical fibres. Phys. Rev. Lett. 96, 010503 (2006).

17. Monroe, C. Quantum information processing with atoms and photons. Nature 416, 238-246 (2002).

18. Grangier, P., Sanders, B. \& Vuckovic, J. (eds) Focus issue on single photons on demand New J. Phys. 6, $85-100 ; 129 ; 163$ (2004).

19. Santori, C., Fattal, D., Vučković, J., Solomon, G. S. \& Yamamoto, Y. Indistinguishable photons from a single-photon device. Nature 419, 594-597 (2002).

20. Legero, T., Wilk, T., Hennrich, M., Rempe, G. \& Kuhn, A. Quantum beat of two single photons. Phys. Rev. Lett. 93, 070503 (2004).

21. Beugnon, J. et al. Quantum interference between two single photons emitted by independently trapped atoms. Nature 440, 779-782 (2006).

22. Press, D. et al. Photon antibunching from a single quantum dot-microcavity system in the strong coupling regime. Preprint at $<$ http://www.arxiv.org/quant-ph/0609193> (2006).

23. Hennrich, M., Legero, T., Kuhn, A. \& Rempe, G. Vacuum-stimulated Raman scattering based on adiabatic passage in a high-finesse optical cavity. Phys. Rev. Lett. 85, 4872-4875 (2000).

24. Keller, M., Lange, B., Hayasaka, K., Lange, W. \& Walther, H. Continuous generation of single photons with controlled waveform in an ion-trap cavity system. Nature 431, 1075-1078 (2004).

25. Wilk, T., Webster, S. C., Specht, H. P., Rempe, G. \& Kuhn, A. Polarization-controlled single photons. Phys. Rev. Lett. 98, 063601 (2007).

26. Nußmann, S. et al. Submicron positioning of single atoms in a microcavity. Phys. Rev. Lett. 95, 173602 (2005).

27. Kuhn, A., Hennrich, M., Bondo, T. \& Rempe, G. Controlled generation of single photons from a strongly coupled atom-cavity system. Appl. Phys. B 69, 373-377 (1999).

Acknowledgements

This work was supported by the Deutsche Forschungsgemeinschaft (SFB 631 and Research Unit 635) and the European Union (IST (SCALA) and IHP (CONQUEST) programs).

Correspondence and requests for materials should be addressed to G.R.

Competing financial interests

The authors declare no competing financial interests.

Reprints and permission information is available online at http://npg.nature.com/reprintsandpermissions/ 